\title{
Wireless Video Transmission: A Single Layer Distortion Optimal Approach
}

\author{
Negar Nejati Homayoun Yousefi'zadeh Hamid Jafarkhani \\ Department of EECS \\ University of California, Irvine \\ [nnejati,hyousefi,hamidj] @uci.edu
}

\begin{abstract}
In this paper, we introduce an analytical expression for the expected distortion of a single layer encoded video bit-stream. Based on the expected distortion model, we propose a distortionoptimal Unequal Error Protection (UEP) technique to transmit such bit-stream over a wireless tandem channel. The proposed method allocates the total transmission budget unequally to different frames of a video bit-stream in order to protect the bit-stream against both bit errors caused by fading and packet erasures caused by network buffering. We compare this technique with another UEP technique as well as a one-dimension equal length protection technique. The evaluation results for different choices of packet sizes, available budgets, and channel conditions show that the proposed method outperforms the other alternative schemes.
\end{abstract}

\section{INTRODUCTION}

Despite improvements in wireless network infrastructure and transmission techniques of multimedia content, there are still many challenges in providing an acceptable level of Quality of Service (QoS) for video delivery over wireless channels. Several transmission techniques have been proposed to improve the quality of reconstructed video delivered over packet erasure only channels. Often times, UEP methods protect different parts of an encoded video bitstream according to their importance therefore offering an allocation of system resources is necessary for minimizing the expected distortion of a such bitstream delivered over a transmission medium. Further, researchers have applied UEP to different layers of scalable coding [1] [2]. For example, a combination of Fine Granular Scalability (FGS) and UEP techniques has been proposed in [3] for video transmission over a packet erasure only channel. The results obtained in [3] show an improvement in quality of reconstructed video compared to a single layer protection scheme, even without optimizing Forward Error Correction (FEC) rates. Other techniques protect different types of frames, $I, P$, and $B$ frames according to their importance in order to minimize the expected distortion of a transmitted video sequence over erasure channels [4] [5] [6]. A combination of these two aspects has also been proposed. The authors of [7] have proposed a GOP-Based rate allocation method for scalable video streaming over error-prone networks by utilizing the expected distortion model of [8]. This model is accurate for low bit error rate channels where residual error rate is typically less than $10 \%$.

Wireless channels are identified by temporally correlated tandem loss patterns which appear in the form of bit errors related to fading effects and packet erasures related to network layer buffering. However, the effects of fading related bit errors is not considered in any of the literature works above. Both [9] and [10] used RCPC/CRC and RS product codes for scalable multimedia transmission over wireless channels identified by bit errors 
and packet erasures. In [11], we propose a low complexity UEP technique to optimally protect a progressive FGS bitstream against both bit errors and packet erasures under the assumption that base layer is received error free.

As the main motivation of this work, we differentiate between two video transmission scenarios over wireless tandem channels. The first scenario which is the subject of our earlier work deals with optimally improving the quality of a multi layer continuously progressive video bitstream beyond a given basic quality provided by error free delivery of the base layer. The second scenario which is the subject of our current work makes no assumption about either error free delivery of a base layer or the availability of the progressiveness feature. Thus, we relax our previous assumptions in this paper and propose a low complexity optimal-distortion rate allocation method to optimally protect a single (base) layer encoded video bitstream against bit errors and packet erasures. In this work, we allocate the available transmission budget to the frames of an encoded sequence based on the importance of such frames in the quality of the sequence.

The rest of this paper is organized as follows. In section II, we briefly introduce our channel model which captures both bit errors and packet erasures. Section III, contains the expected distortion model of the received video sequence over a wireless channel. In Section IV, we first introduce some rate-allocation schemes and then formulate an optimization problem based on our distortion model. Section V compares the results of our proposed technique to other alternatives. Finally, we summarize our work in Section VI.

\section{Channel Model}

As indicated earlier, both packet erasures and bit errors need to be captured in order to properly model a wireless channel. While bit errors are produced by the wireless transmission medium, packet loss is caused by network impairments such as congestion. To capture the effects of bit errors and packet erasures in our study [12], we use a two-state Gilbert-Elliott (GE) Markov chain and another independent Gilbert (G) Markov chain, respectively. A two-state GE chain with GOOD and BAD states is uniquely identified by a pair of transitioning probabilities and a pair of per state error probabilities. Bit errors caused by a fading channel are modeled by a GE chain in which $\gamma$ is the self transitioning probability for GOOD state and $\beta$ is the probability of self transitioning for BAD state. Per state error probabilities are specified as $\epsilon_{G}$ and $\epsilon_{B}$. The $\mathrm{G}$ chain is similar to the GE chain except that GOOD and BAD states have trivial error probabilities. In the GE model, the probability of $n$ errors in $k$ transmissions is calculated as

$$
P(n, k)=P(n, k, G)+P(n, k, B),
$$

where $P(n, k, G)$ and $P(n, k, B)$ are the probabilities of having $n$ errors in $k$ transmissions and ending up in GOOD and BAD state, respectively. These probabilities are calculated as

$$
\begin{aligned}
& P(n, k, G)=\left(1-\epsilon_{G}\right) \gamma P(n, k-1, G)+\left(1-\epsilon_{G}\right)(1-\beta) P(n, k-1, B) \\
& +\epsilon_{G} \gamma P(n-1, k-1, G)+\epsilon_{G}(1-\beta) P(n-1, k-1, B),
\end{aligned}
$$

and

$$
\begin{aligned}
& P(n, k, B)=\left(1-\epsilon_{B}\right)(1-\gamma) P(n, k-1, G)+\left(1-\epsilon_{B}\right) \beta P(n, k-1, B) \\
& +\epsilon_{B}(1-\gamma) P(n-1, k-1, G)+\epsilon_{B} \beta P(n-1, k-1, B)
\end{aligned}
$$


where $P(n, 0, G)=P(n, 0, B)=0$ for $n \neq 0$ and initial conditions are

$$
P(0,0, G)=\frac{1-\beta}{1-\gamma-1-\beta}, P(0,0, B)=\frac{1-\gamma}{1-\gamma-1-\beta},
$$

According to [13], the probability of symbol error for the GE chain depends on the average received signal-to-noise ratio, the modulation scheme, the number of signal points in the modulation constellation $M$, the number of transmit/receive antennas, and the utilized coding technique. The details of calculating the probability of symbol error in each state can be found in [11].

We model packet erasures with a $\mathrm{G}$ chain. The $\mathrm{G}$ chain operates similar to the $\mathrm{GE}$ chain with $\epsilon_{G}=0$ and $\epsilon_{B}=1$. However, we note that the probabilities of error are related to network buffering dynamics as opposed to fading statistics. With $P_{e}$ defined as the average probability of packet erasure, $\beta$ is calculated as follows:

$$
\beta=2-\gamma-\frac{(1-\gamma)}{P_{e}},
$$

From (4), we can see that increasing $P_{e}$ will increase $\beta$ and consequently the probability of packet erasure.

\section{Proposed Expected Distortion Model}

In this section, we propose an analytical model for the expected distortion of a single layer decoded video bitstream transmitted over a wireless channel.

The expected distortion of such sequence depends on the expected distortion of each GOP of the sequence. When a frame is lost, the error concealment method of the decoder reconstructs the lost frame using previous frames thereby causing error propagation. Intracoded frames are used to prevent the effects of error propagation from one GOP to the next. If an intra-coded frame is lost then the previous frame which belongs to the previous GOP will be used to reconstruct it. This means that any error in the previous GOP will be propagate to the current GOP. Therefore when error concealment method is utilized at the decoder side, distortion of GOPs can not be considered as an independent units unless the intra-frames are received correctly. In this paper, we assume that by providing sufficient error protection to the intra-coded frames, GOPs can be treated independently and therefore the expected distortion of the sequence is calculated as the summation of the expected distortion of all GOPs of the sequence as:

$$
\epsilon_{d}=\sum_{i=1}^{G} \epsilon_{d}(i)
$$

where $G$ is the number of GOPs in the sequence and $\epsilon_{d}(i)$ represents the expected distortion of the $i$-th GOP of the video sequence. The expected distortion of a GOP is represented using table $I$ in which it is assumed that the probability of receiving an $I$ frame is not less than a threshold. This threshold is identified by protecting intra-coded frames against channel errors. In table I, each row indicates the pattern of the received frames in the GOP and also the distortion of GOP associated with this pattern after using the error concealment technique. Assuming $n$ is the size of GOP, the total number of rows is $2^{n-1}$. The first row 
TABLE I

THE DISTORTION OF A GOP BASED ON THE PATTERN OF RECEIVED FRAMES

\begin{tabular}{|c|c|c|c|c|}
\hline Frame 1 & Frame 2 & $\ldots$ & Frame n-1 & Distortion \\
\hline$P(0,1)$ & $P(0,2)$ & $\ldots$ & $P(0, n-1)$ & $d_{0}$ \\
\hline$P(0,1)$ & $P(0,2)$ & $\ldots$ & $P(1, n-1)$ & $d_{1}$ \\
\hline & & & $\ldots$ & \\
\hline$P(1,1)$ & $P(1,2)$ & $\ldots$ & $P(1, n-1)$ & $d_{2^{n-1}-1}$ \\
\hline
\end{tabular}

indecates the case where all the $n$ frames are lost and the last row shows the case that all the $n$ frames of the GOP are received correctly. With $d_{i}$ indicating the distortion of GOP associated with the $i$-th row, the probability of receiving frame $j$ without error $P(1, j)$ is identified as:

$$
P(1, j)=\prod_{k=0}^{N_{j}-1} P_{p c k}(1, k)
$$

where $N_{j}$ defines the number of packets for transmitting frame $j$ and $P_{p c k}(1, k)$ represents the probability of receiving the $k$-th packet of frame $j$ free of error. $P_{p c k}(1, k)$ is calculated as:

$$
P_{p c k}(1, k)=\left(1-P_{e s}\right)^{L}
$$

where $L$ is the number of symbols in each packet. As a matter of convenience and without losing generality, we assume that each symbol is one byte long and $P_{e s}$ is the probability of symbol error calculated using the channel model. $P(0, j)=1-P(1, j)$ is the probability of loss for frame $j$. We use $P^{i, j}$ to refer to the probability of the $j$-th frame in the $i$-th row of Table I. We note that $P^{i, j}$ is either $P(0, j)$ or $P(1, j)$ depending on the loss pattern of row $i$ in the probability table of that GOP. Using the introduced table, the expected distortion of GOP can be defined as follows:

$$
\epsilon_{d}(i)=\sum_{k=1}^{2^{n-1}-1} \prod_{j=1}^{n-1} P^{k, j} d_{k}
$$

Utilizing two different threshold values, Fig. 1 compares sample results of the proposed analytical model of this section with experimental distortion results of the Foreman sequence in different channel conditions. While the sample results are reported for Foreman sequence, similar patterns are observed in the case of other sequences.

As shown in the figures, the analytical model cannot closely match the experimental results for lower threshold values, specially, in the case of channels identified with high error rates. We note that the latter is due to the fact that the assumption of GOPs being independent is no longer valid in such cases.

\section{Optimized TRAnsmission Method}

The overall minimum expected distortion for the whole bitstream can be achieved by minimizing the expected distortion of each GOP, separately. Each frame in a GOP has a 


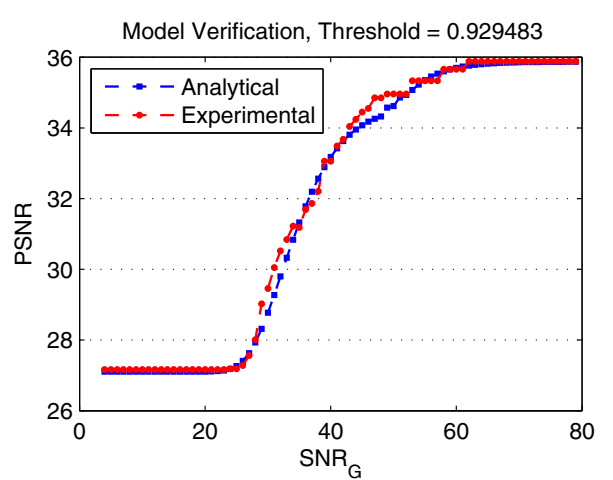

(a)

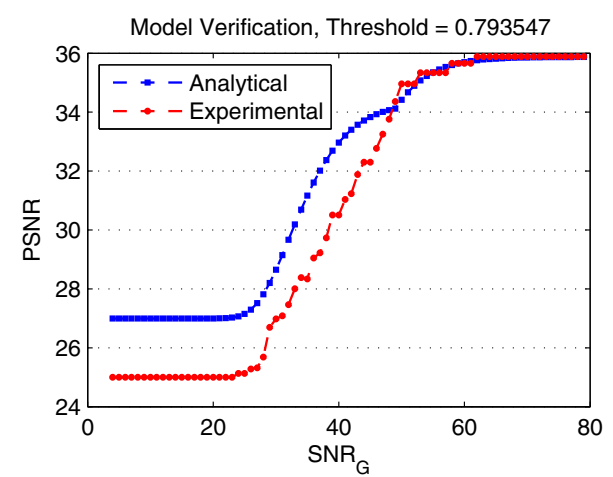

(b)

Fig. 1. A comparison of analytical and experimental distortion results of Foreman sequence with a threshold value of (a) 0.929483 and (b) 0.793547 .

different effect on the distortion of the GOP depending on its encoding type and location in the GOP. This constitutes a rate allocation problem.

In this section, we discuss three alternatives of protecting a video bitstream against channel loss effects including two optimization techniques capturing the rate allocation problem described above. First, we explain the so-called Equal Length Protection (ELP) technique. Next, we introduce a simple linear rate allocation technique based on the distortion caused by the loss of each GOP and each frame. We refer to such technique as Linear Distortion Optimal (LDO) technique. Finally, we formulate our constrained optimization problem to which we refer to as Optimal Single Layer (OSL). We reiterate that OSL is associated with the transmission of video over a channel characterized by tandem loss. While the ELP technique is used as a baseline, the two optimization techniques are all targeted at minimizing the overall expected distortion of a video bitstream and are subject to a total transmission budget. To provide same transmission conditions for all of the transmission methods, we assume that the intra-coded frames are protected with enough amount of parities to achieve the threshold of our model, in the same way as the OSL method. Reed-Solomon (RS) coding is the FEC technique utilized in all techniques.

\section{A. The ELP Technique}

In this technique, the total parity budget is distributed equally among all the inter-coded frames. In other words, the amount of parity allocated to each frame is calculated independent of the length of that frame and the effect of that frame on the total distortion of the GOP. In ELP, the packet length is fixed and set equal to the RS code word. This one dimensional code, protects each packet against the bit errors with a fix number of parities and cannot support the packets against packet erasures.

\section{B. The LDO Technique}

As it was mentioned previously, different types and locations of frames have different effects on the distortion of a GOP. LDO is a simple linear rate allocation technique for transmitting the encoded video bitstream over a tandem channel. LDO assigns parity budget to each frame based on the ratio of the distortion that its loss will cause to the total 
distortion of that GOP. This rate allocation can be formulated as follows:

$$
\begin{aligned}
\text { Budget }_{\text {frame }}\left(i_{j}\right) & =\operatorname{Budget}_{G O P}(i) \times \frac{\operatorname{dist}_{\text {frame }}\left(i_{j}\right)}{\operatorname{dist}_{G O P}(i)} \\
\operatorname{dist}_{G O P}(i) & =\sum_{j=0}^{n_{i}-1} \operatorname{dist}_{\text {frame }}\left(i_{j}\right),
\end{aligned}
$$

where Budget $_{\text {frame }}\left(i_{j}\right)$ represents the budget of frame $j$ in GOP $i$, $\operatorname{dist}_{\text {frame }}\left(i_{j}\right)$ is the distortion of GOP $i$ when frame $j$ of the GOP is lost, $\operatorname{dist}_{G O P}(i)$ is the average GOP distortion caused by any single frame lost, and $n_{i}$ is the size of GOP $i$. The budget of GOP $i$ can also be calculated utilizing the same approach:

$$
\begin{aligned}
\text { Budget }_{G O P}(i) & =\text { total_Budget } \times \frac{\text { dist }_{G O P}(i)}{\text { total_dist }_{-}}, \\
\text {total_dist } & =\sum_{i=0}^{G-1} \operatorname{dist}_{G O P}(i),
\end{aligned}
$$

where $G$ is the number of GOPs in the whole video sequence and total_Budget is the maximum transmission budget depending on the available bandwidth. To implement this technique, we calculate the required distortions offline. Error is inserted manually into the specific location of the encoded bitstream. Then, it is decoded and its distortion is calculated.

\section{The OSL Technique}

As shown in the previous section, the expected distortion of a single layer video bitstream can be modeled using (5). We assume that the encoded video bitstream is packetized with a fixed packet size and each frame is protected with a one-dimensional RS code. We note that our coding scheme interleaves the symbols to better cope with the temporally correlated loss observed over the tandem channels of interest. Fig. 2 illustrates our proposed OSL technique. In the figure, $N$ is the length of packets, $L$ is the number of packets containing

\begin{tabular}{|c|c|c|c|c|c|c|}
\hline 1 & 5 & 9 & 13 & 17 & 21 & 25 \\
\hline 2 & 6 & 10 & 14 & 18 & 22 & 26 \\
\hline 3 & 7 & 11 & 15 & 19 & 23 & 27 \\
\hline 4 & 8 & 12 & 16 & 20 & 24 & 28 \\
\hline \\
\hline
\end{tabular}

Fig. 2. An illustration of the proposed OSL technique.

the data of encoded video frame, and $K$ is the number of channel coding symbols in each column. The total budget for transmitting data and parity of an inter-coded frame is equal to $N *(L+K)$ and the budget of parity for transmitting the intra-coded frame is calculated by using the threshold defined by the analytical model. Therefore, the optimization steps for GOP $i$ include: 
- Finding the parity budget for the $I$ frame as

$$
P(1, I) \geq \text { Threshold }
$$

- Finding the parity budget for the other frames of the GOP as

$$
\begin{gathered}
\underset{\mathbf{R}_{\mathbf{f}_{1}}, \mathbf{R}_{\mathbf{f}_{2}}, \ldots, \mathbf{R}_{\mathbf{f}_{\mathbf{n}-1}}}{\min } \epsilon_{d}(i) \\
\text { subject to : } 0 \leq \sum_{k=1}^{n-1} R_{f_{k}}<\text { Budget }
\end{gathered}
$$

where $\epsilon_{d}(i)$ indicates the expected distortion of the $i$ GOP in the video sequence, $R_{f_{k}}$ represents the rate allocated to the $k$-th frame $f_{k}$, and $n$ shows the size of GOP. As mentioned in Section III, $\epsilon_{d}(i)$, the expected distortion of GOP can be defined as:

$$
\epsilon_{d}(i)=\sum_{i=1}^{2^{n-1}-1} \prod_{j=1}^{n-1} P_{r e c}^{i, j} d_{i}
$$

where $n$ shows the number of frames in the GOP. If the $j$-th frame of the $i$-th row in the probability table of frame $j$ is marked as recovered, then $P_{r e c}^{i, j}$ can be represented by $P_{\text {rec }}(1, j)$ which is the probability of recovery for the $j$-th frame. Otherwise, it would be equal to $P_{r e c}(0, j)=1-P_{r e c}(1, j)$. An RS code with $K$ parity symbols can successfully reconstruct the data of each codeword if the number of symbol errors $N_{\text {err }}$ and the number of symbol erasures $N_{\text {ers }}$ satisfy $2 N_{\text {err }}+N_{\text {ers }} \leq K$. Therefore considering the transmission technique of Fig. 2, $P_{r e c}(1, j)$ can be defined based on $P_{c_{j}}$, the probability of recovery of each column of frame $j$, as follows:

$$
\begin{aligned}
& P_{\text {rec }}(1, j)=\left(P_{c_{j}}\right)^{N} \\
& P_{c_{j}}=\sum_{l=0}^{K_{j}} P\left(N_{\text {err }} \leq\left\lfloor\frac{K_{j}-l}{2}\right\rfloor \mid N_{\text {ers }}=l\right) P_{\text {ers }}\left(M_{j}, l\right), \\
& P\left(N_{\text {err }} \leq\left\lfloor\frac{K_{j}-l}{2}\right\rfloor \mid N_{\text {ers }}=l\right)=\sum_{k=0}^{\left\lfloor\frac{K_{j}-l}{2}\right\rfloor} P\left(N_{\text {err }}=k \mid N_{\text {ers }}=l\right), \\
& P\left(N_{\text {err }}=k \mid N_{\text {ers }}=l\right)=\left(\begin{array}{c}
M_{j}-l \\
k
\end{array}\right) P_{e s}^{k}\left(1-P_{e s}\right)^{M-l-k},
\end{aligned}
$$

where $P_{e s}$ is the probability of symbol error, $K_{j}$ is the number of parity symbols for each column of frame $j$ and $M_{j}=L_{j}+K_{j}$ is the number of packets transmitted for that frame. It is important to note that $P_{r e c}(1, j)$ considers the effects of both symbol errors and symbol erasures.

Considering the limitations of the bandwidth for the channel we assume that the acceptable parity budget for the whole sequence is between $0 \%$ to $25 \%$. Under this assumption, this optimization problem can be solved relying on an intelligent search strategy described 
below. To describe our proposed search strategy, we note that the number of possible allocations can be significantly reduced considering the fact that the earlier frames in GOP have a higher priority. This will add a constraint to our optimization problem as follows:

$$
K_{0} \geq K_{1} \geq K_{2} \geq \ldots \geq K_{n-1}
$$

where $K_{0}$ associates with the parity of the $I$ frame, $K_{i}$ is the number of parity packets allocated to the frame $i$, and $n$ is the number of frames in each GOP. For the case of GOP size equal to four, there are three inter-coded frames in the GOP. We use $T$ to represent the number of total parity packets for that GOP which is the summation of $K_{1}, K_{2}$, and $K_{3}$ in the latter case. In the case of full search, the number of possible parity packet allocations to the first packet is $T$ and for each case of $K_{1}$, the possible parity packet allocations among the second and third frames would be $T-K_{1}+1$. Therefore, the total number of parity packet allocations can be calculated as follows:

$$
\sum_{K_{1}=0}^{T}\left(T-K_{1}+1\right)=\frac{(T+1)(T+2)}{2}
$$

By constraining our optimization problem with (20), the number of possible parity packet allocations for $K_{3}$ will be $\left\lfloor\frac{T}{3}\right\rfloor$ and for each case of $K_{3}$ there are $\left(\left\lfloor\frac{T-K_{3}}{2}\right\rfloor+1-K_{3}\right)$ possible parity packet allocations for both $K_{1}$ and $K_{2}$. Thus, the number of possible parity packet allocations will be calculated as follows:

$$
\sum_{K_{3}=0}^{\left\lfloor\frac{T}{3}\right\rfloor}\left(\left\lfloor\frac{T-K_{3}}{2}\right\rfloor+1-K_{3}\right)
$$

which, in the worst case, is equal to $\frac{(T+1)(T+5)}{12}$ Therefore the ratio of the possible allocations in this last case to the full search case is $\frac{(T+5)}{6(T+2)}$. For large $T$, this ratio will be equal to $1 / 6$ which shows reducing the number of possible parity packet allocations up to $83 \%$.

In this optimization problem, we assume that the total transmission budget $M_{G O P}(i)$ of GOP $i$ is known. To find this budget, we use an offline approach similar to the one utilized by the LDO technique. More specifically, we find the rate-distortion curve of the decoder first. Then, we calculate the probability of recovering each frame under the given channel condition and the given parity for each column of that frame. Using (15), the distortion of GOPs are calculated. This process is repeated for all possibilities of the parity allocation limited by the total amount of parity for each GOP. Having identified all allocations and associated distortions, the optimal parity allocation in the GOP and minimum distortion of GOP in the given channel condition is calculated.

\section{COMPARISON RESUlts}

In this section, we provide the comparison results of our proposed technique OSL with those of ELP, and LDO techniques for different channel conditions and total budgets. In our experiments, we transmit over a channel introducing both bit errors and packet erasures. The video codec used in this work is MoMuSys [14] [15] implementing MPEG4 standard. The GE chain is used to capture temporally correlated pattern of bit errors. For the GE 
chain, we apply transitioning probabilities of $\gamma=0.99875$ and $\beta=0.875$ associated with average burst lengths of 800 and 8 bits. We choose an SNR range of $[4,60] \mathrm{dB}$ for GOOD state of the GE chain and set $S N R_{G}=10 S N R_{B}$ to differentiate between the two states. An independent $\mathrm{G}$ chain is used to capture temporally correlated pattern of packet erasures. The $\mathrm{G}$ chain has the same $\gamma$ as the GE chain and its $\beta$ parameter changes in the range of $[0.87625,0.995]$. In this work, we also use robust header compression [16] to compress the RTP/UDP/IP header into 3 bytes. This will help reduce the overhead of packetizing the video bitstream. From among the set of different sequences utilized in our experiments, we only report the results of our experiments with Foreman sequence. The total budget and packet size are fixed for all of the transmission techniques. Applying a total parity budget equal to $18 \%$ of the size of the original video bitstream and a packet size of 64 bytes, Fig. 3(a) illustrates the results of OSL technique applied to the single layer encoded bitstream of Foreman sequence in qcif format. Fig. 3(b) shows the quality improvement of the received video sequence using the proposed OSL technique when the total transmission budget is increased.
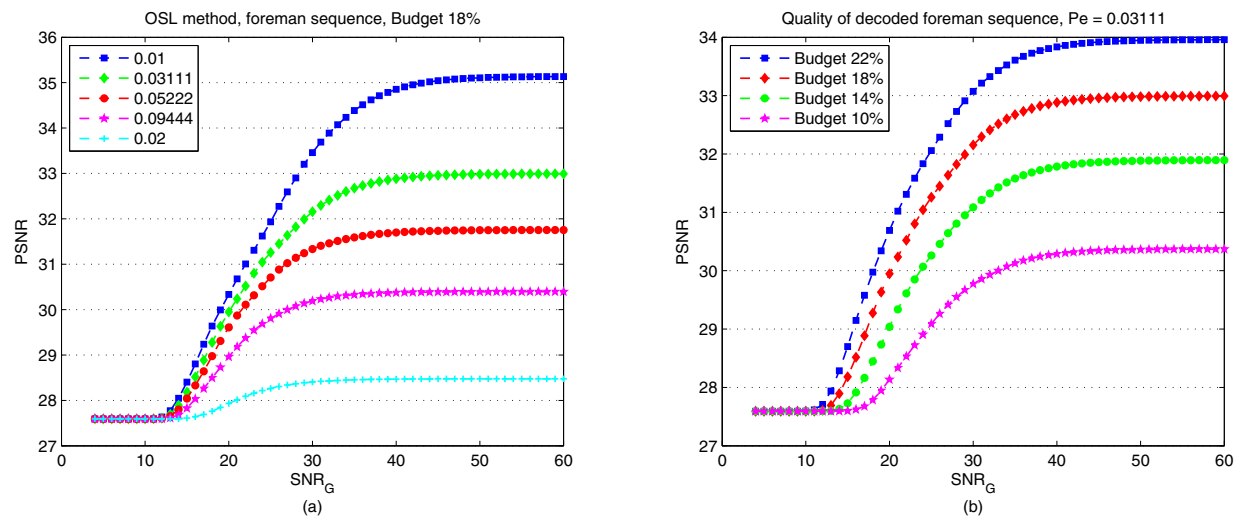

Fig. 3. The results of OSL technique for (a) different channel conditions when a parity budget equal to $18 \%$ of the size of the original video bitstream is utilized, (b) different parity budgets and different bit error conditions.

The comparison results of OSL, LDO and ELP techniques are shown in Fig. 4(a) and Fig. 4(b). In Fig. 4(a) the total transmission budget is fixed and equal to $114 \%$ of the original bitstream size. However in Fig. 4(b), the results are shown for different budgets while the packet loss probability of the channel is assumed to be fixed. The results show that our proposed OSL technique can improve the quality of received video specially in high packet erasure rate channels.

\section{CONCLUSION}

In this paper, we proposed an analytical model for the expected distortion of a single layer encoded video bitstream. Utilizing our distortion model and MoMuSys video codec implementing MPEG4 standard, we formulated and solved a distortion-optimal problem of video transmission over a tandem channel introducing fading related bit errors and network buffering related packet erasures. We compared the performance of our proposed distortion-optimal method to which we referred as OSL with an equal error protection method referred to as ELP, and an unequal error protection method called LDO. Our results illustrated that our proposed method outperforms other methods specially for low quality channel conditions. 

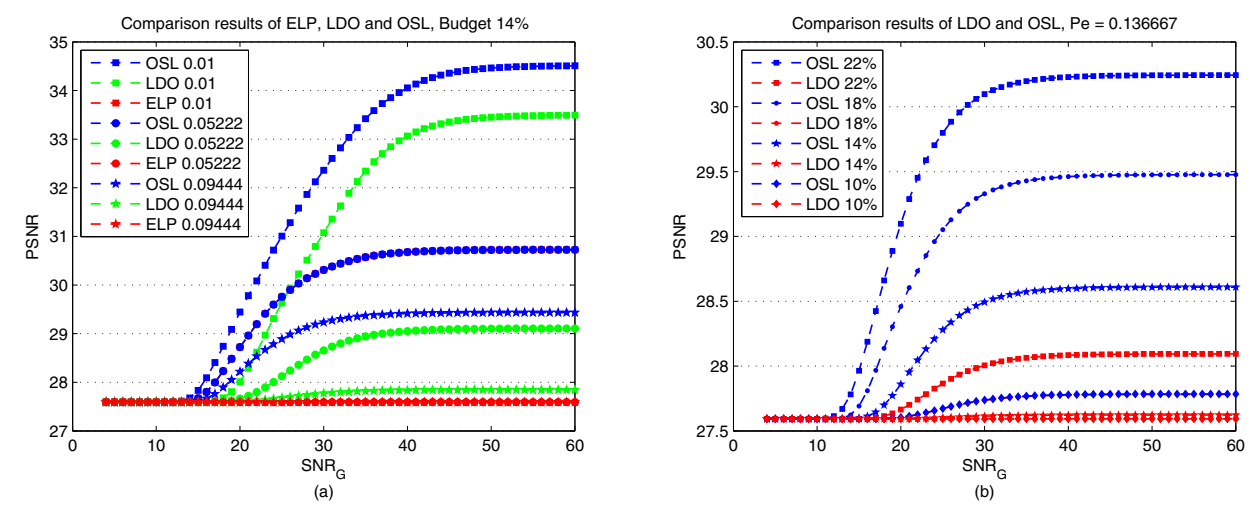

Fig. 4. The comparison results of (a) OSL, LDO and ELP techniques for different channel conditions when parity budget equal to $14 \%$ of the size of the original video bitstream is utilized, (b) OSL and LDO techniques for different budgets and different bit error conditions.

\section{REFERENCES}

[1] M. Schaar and H. Radha, "Unequal Packet Loss Resilience for Fine-Granular-Scalability Video," IEEE Trans. on Multimedia, Dec 2001.

[2] F. Zhai, Y. Eisenberg, T. N. Pappas, R. Berry, and A. K. Katsaggelos, "Rate-Distortion Optimization Product Code Forward Error Correction for Video Transmission over IP-Ba sed Wireless Networks," in Proc. IEEE ICASSP, 2004.

[3] T. Schierl, H. Schwarz, D. Marpe, and T. Wiegand, "Wireless Broadcasting Using the Scalable Extension of H.264/AVC," in Proc. IEEE ICME, 2005.

[4] T. Fang and L. Chau, "A Novel Unequal Error Protection Approach for Error Resilient Video Transmission," in Proc. IEEE Int. Symp. Circuits System, May 2005.

[5] C. Haung and S. Liang, "Unequal Error Protection for MPEG-2 Video Transmission over Wireless Channels," Signal Processing Image Communications, Jan 2004.

[6] X. Y. et al., "Unequal Loss Protection for Robust Transmission of Motion Compensated Video over the Internet," Signal Processing Image Communications, March 2003.

[7] T. Fang and L. P. Chau, "GOP-Based Channel Rate Allocation using Genetic Algorithm for Scalable Video Streaming over Error-Prone Networks," IEEE Trans. on Image Processing, Jun 2006.

[8] K. Stuhlmuller, N. Farber, M. Link, and B. Girod, "Analysis of Video Transmission over Lossy Channels," IEEE JSAC, Jun 2000.

[9] S. Zhao, Z. Xiong, and X. Wang, "Optimal Resource Allocation for Wireless Video over CDMA Networks," in Proc. IEEE ICME, 2003.

[10] V. Stankovic, R. Hamzaoui, and Z. Xiong, "Joint Product Code Optimization for Scalable Multimedia Transmission over Wireless Channels," in Proc. IEEE ICME, 2002.

[11] N. Nejati, H. Yousefi'zadeh, and H. Jafarkhani, "Wireless Video Transmission: A Distortion-Optimal Approach," in Proc. IEEE DCC, Mar 2008.

[12] H. Yousefi'zadeh, F. Etemadi, and H. Jafarkhani, "Distortion-Optimal Transmission of Progressive Images over Channels with Random Bit Errors and Packet Erasures," in Proc. IEEE DCC, 2004.

[13] H. Yousefi'zadeh, H. Jafarkhani, and M. Moshfeghi, "Power Optimization of Wireless Media Systems with SpaceTime Block Codes," IEEE Trans. on Image Processing, Jul 2004.

[14] "General project information, the momusys web site," Available at http://www.tnt.unihonnover.de/project/eu/momusys/.

[15] "Final Report on Standardisation Activities, MoMuSys Public Deliverable," Available at http://www.tnt.unihonnover.de/project/eu/momusys/doc/mom-docs.html.

[16] S. Casner and V. Jacobson, “Compressing IP/UDP/RTP Headers for Low-Speed Serial Links,” RFC2508, Feb. 1999. 